\title{
Supporting Information \\ Synthesis of Antimony Sulfide Nanotubes with Ultrathin Walls via Gradual Aspect Ratio Control of Nanoribbons
}

\author{
Kang Hyun Park, Jae Won Choi, Hae Jin Kim, Jin Bae Lee, and Seung Uk Son* \\ Department of Chemistry, Sungkyunkwan University, Suwon 440-746, Korea \\ and Korea Basic Science Institute, Daejeon 350-333, Korea
}

\section{Experimental Details}

The SEM image in figure 4 and EDS data in figure 2 were obtained by FE-SEM (JSM6700F). The TEM images were taken using a JEOL 2100F unit operated at 200kV. The samples used for the TEM studies were prepared on carbon-coated copper grids by drop-casting nanomaterials dispersed in hexane without any size selection process. The XPS analysis was performed using an ESCALAB 250 XPS spectrometer made by VG Scientifics Co. Powder XRD patterns were obtained on a Rigaku Max-2200 with filtered $\mathrm{Cu}_{\mathrm{ka}}$ radiation. Antimony chloride, sulfur, and oleylamine were purchased Aldrich Co. and used without additional purification.

General procedure used for the preparation of the nanotubes: Under argon in flame-dried $10 \mathrm{~mL}$ glassware, 130 $\mathrm{mg}(0.57 \mathrm{mmol})$ of antimony chloride was dissolved in well-dried oleylamine $(2 \mathrm{~mL})$ at room temperature by sonication. This solution had a pale yellow color. At room temperature, $32 \mathrm{mg}(1.00 \mathrm{mmol})$ of sulfur powder was dissolved in oleylamine $(7 \mathrm{~mL})$. These two solutions were combined in flame-dried $50 \mathrm{~mL}$ Schlenk tube under argon and became reddish yellow. The temperature of reaction mixture was slowly $\left(1.67^{\circ} \mathrm{C} / \mathrm{min}\right)$ increased to $175^{\circ} \mathrm{C}$. Then, the reaction mixture was stirred for an additional 2 hours at this temperature. The color of solution gradually turned brownish black. After cooling to room temperature, the reaction mixture was poured into excess methanol. Brownish precipitates were collected by centrifugation and washed with methanol several times and dried under vacuum.

Figure S1. Magnified TEM images (a-c) of figure 3 in text and additional TEM image (d) of figure 3a.
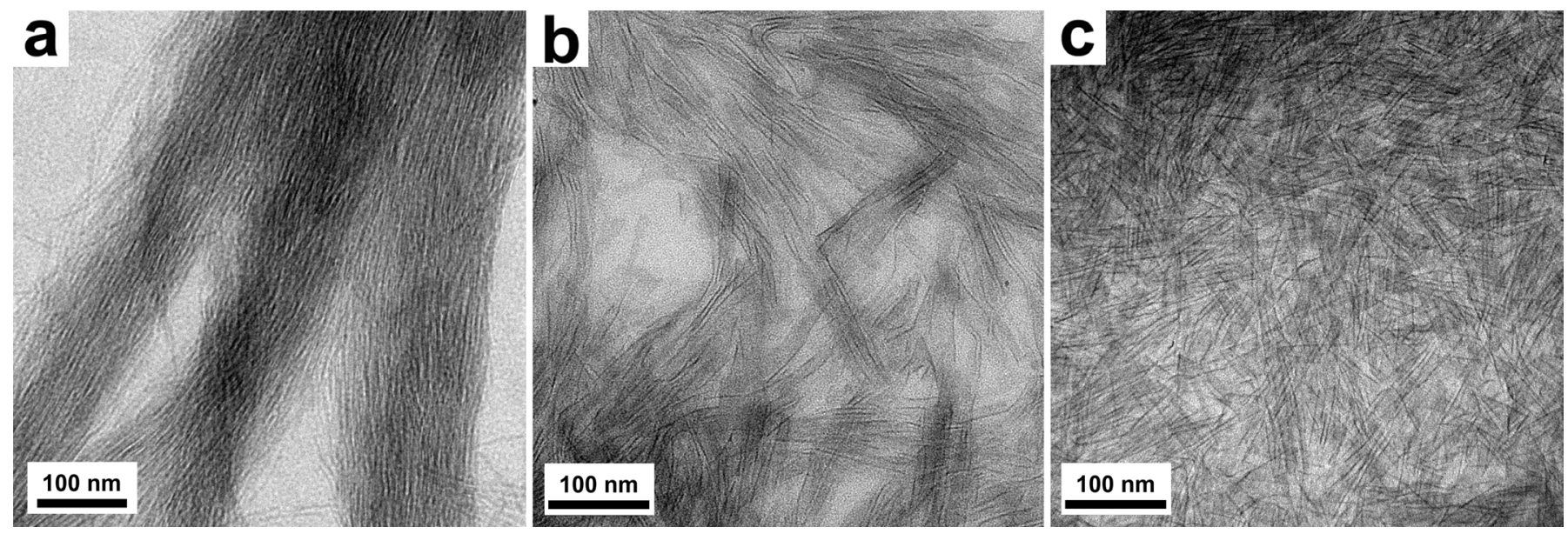


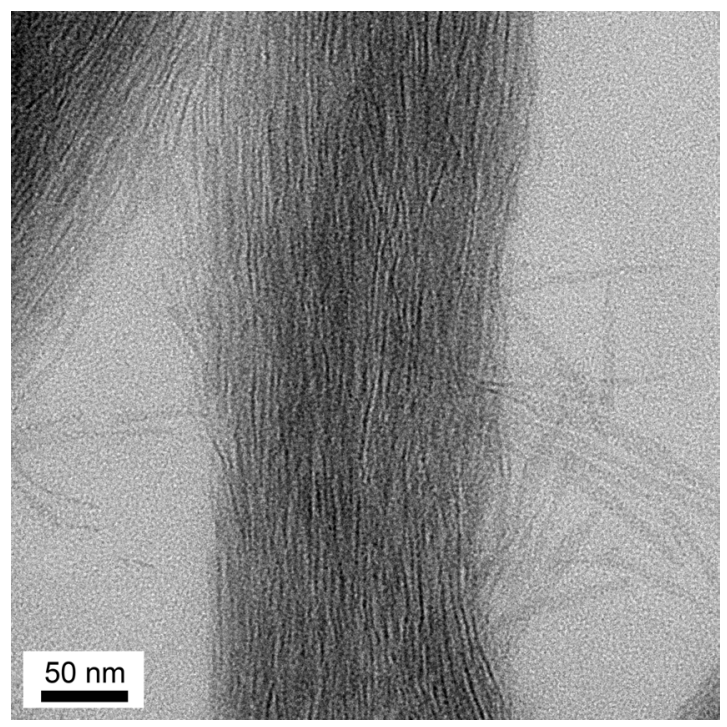

(d)

Figure S2. Time-chasing TEM study: TEM images of nanomaterials obtained just after the reaction temperature reached at $175^{\circ} \mathrm{C}$ (a) and after 30 min later (b).

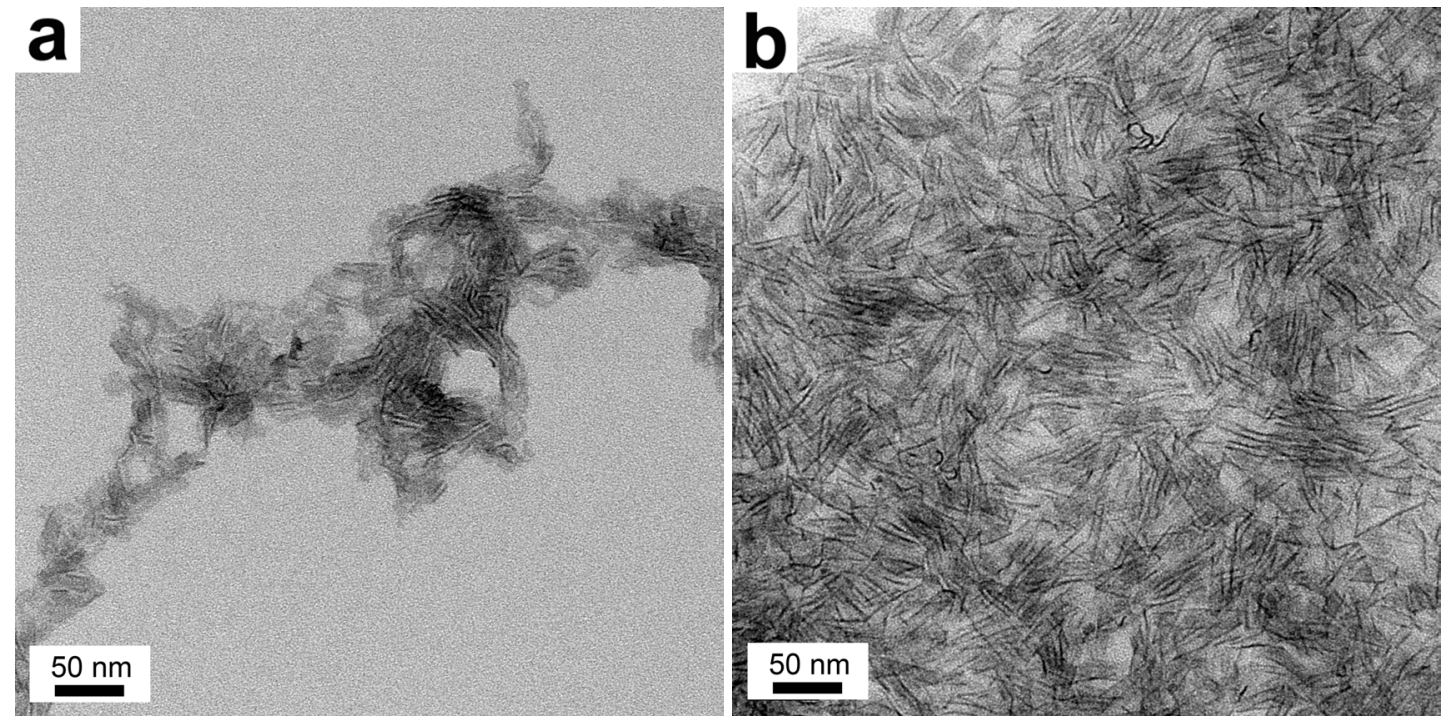

